\title{
Tratamento cirúrgico do câncer colorretal: série histórica de três anos de um serviço em Salvador, Bahia
}

\author{
Surgical treatment of colorectal cancer: three-year historical series of a health \\ service in Savador, Bahia, Brazil
}

\author{
CARLOS RAMON SILVEIRA MENDES ${ }^{1}$; RICARDO AGUIAR SAPUCAIA ${ }^{1}$; \\ LUCIANO SANTANA DE MIRANDA FERREIRA ${ }^{2}$
}

${ }^{1}$ Médico assistente do Serviço de Coloproctologia do Hospital Santa Izabel - Salvador (BA), Brasil. ${ }^{2}$ Chefe do Serviço de Coloproctologia do Hospital Santa Izabel - Salvador (BA), Brasil.

MENDES CRS; SAPUCAIA RA; FERREIRA LSDM. Tratamento cirúrgico do câncer colorretal: série histórica de três anos de um serviço em Salvador, Bahia. Rev bras Coloproct, 2011;30(4): 440-443.

RESUMO: O reduzido número de registro de câncer colorretal no Brasil tem demonstrado a dificuldade para o conhecimento da epidemiologia dessa patologia, levando a um mau planejamento dos governos para o diagnóstico precoce. O objetivo desse trabalho foi relatar a casuística de um serviço, em Salvador (BA), durante um período de três anos e compará-la com a literatura nacional. Os dados obtidos foram analisados, sendo observada semelhança com a literatura brasileira. Ocorreu maior incidência dessa neoplasia entre indivíduos do sexo feminino (53,7\%), com média de idade de 57 anos ao diagnóstico. Nota-se estádio III em 40,4\% dos pacientes, sendo a retossigmoidectomia a cirurgia mais realizada (54\%). Este estudo demonstrou um conhecimento epidemiológico da população baiana, na qual foi observado um atraso do diagnóstico da doença, bem como a necessidade de investimentos na descoberta precoce.

Descritores: Neoplasias colorretais/epidemiologia. Neoplasias.

\section{INTRODUÇÃO}

O câncer colorretal é um importante problema de saúde da população. É a quarta neoplasia maligna mais incidente no Brasil, sendo a quinta causa de morte por câncer em homens e a quarta em mulheres. A carência de dados acerca desta patologia na Bahia dificulta o conhecimento e a correlação com os demais Estados do país.

\section{OBJETIVO}

Demonstrar e analisar a casuística de uma equipe cirúrgica com 66 pacientes portadores de neoplasia colorretal submetidos a tratamento cirúrgico ao longo de três anos.

\section{MATERIAL E MÉTODOS}

Estudo retrospectivo e descritivo realizado no Hospital Santa Izabel em Salvador (BA) após levantamento do banco de dados de uma equipe no período de junho de 2006 a junho de 2009.

\section{RESULTADOS}

O câncer colorretal foi mais frequente entre mulheres $(n=36 ; 53,7 \%)$ do que entre homens $(n=31$; 46,3\%) (Figura 1). A média de idade foi de 57 anos, havendo 13 pacientes (19,7\%) com menos de 40 anos (Figura 2). A localização mais comum foi o retossigmoide ( $\mathrm{n}=35 ; 52,2 \%)$ seguido pelo cólon esquerdo e reto baixo $(n=11 ; 16,4 \%$ cada) e cólon direito $(n=8$;

Trabalho realizado na Santa Casa de Misericórdia da Bahia, Hospital Santa Izabel - Salvador (BA), Brasil..

Recebido em 03/08/2010

Aceito para publicação em 10/09/2010 
11,9\%), com dois casos de tumor sincrônico 3\% (Figura 3). A maioria dos casos era adenocarcinoma moderadamente diferenciado ( $\mathrm{n}=39$; $59 \%$ ), sendo 2 casos de adenocarcinoma mucoprodutor com células em anel de sinete. Houve predomínio de tumores estádio pT3 ( $n=34 ; 51 \%)$ e pN0 ( $n=39 ; 59 \%)$; foram estádio III ( $n=27 ; 40,4 \%)$, estádio II ( $n=25 ; 37,3 \%)$ e estádio I

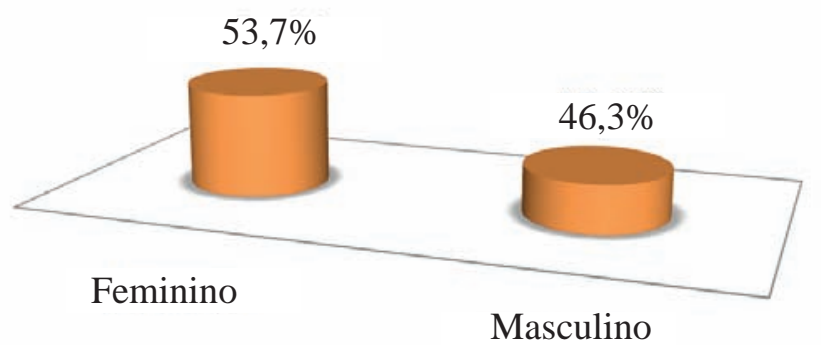

Figura 1 - Distribuição por sexo

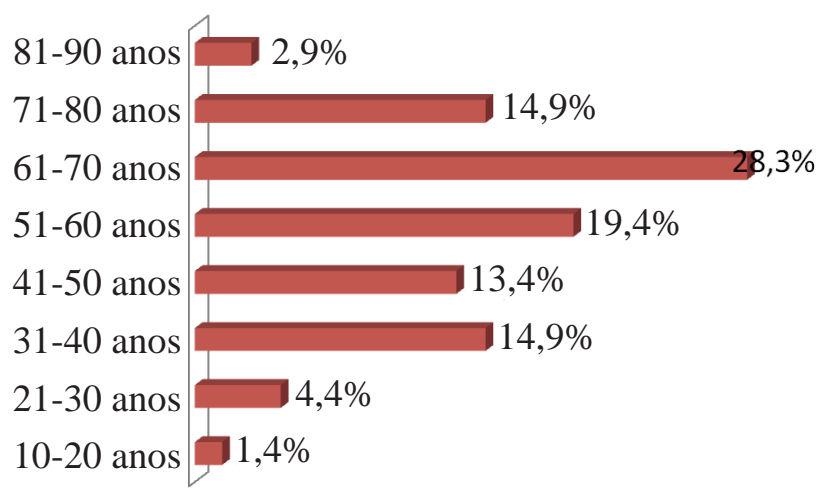

Figura 2 - Distribuição por faixa etária

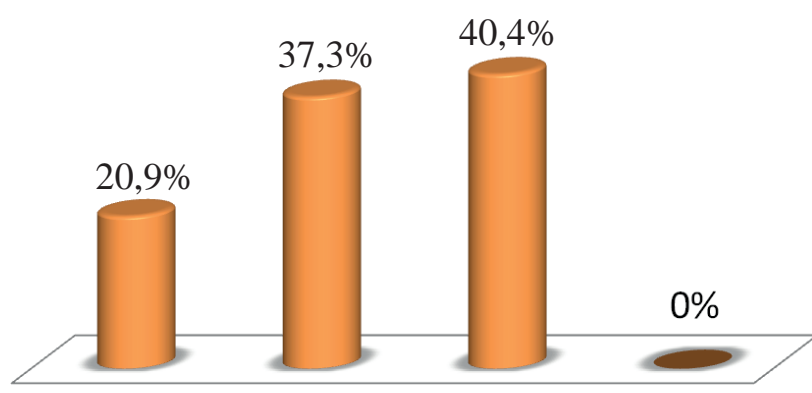

Estádio I Estádio II Estádio III Estádio IV

Figura 3 - Estadiamento anatomopatológico
( $\mathrm{n}=14 ; 20,9 \%$ ) (Figura 4). A média de linfonodos ressecados foi 19 gânglios por peça, apresentando invasão em 14 pacientes.

\section{DISCUSSÃO}

Analisando os dados apresentados e comparando com a literatura, podemos observar, no trabalho publicado por Priolli et al., uma incidência no sexo masculino de 45,1\% ${ }^{1-4}$. Fato semelhante foi visto por Joinvile, Pinho et al. ${ }^{5}$, com incidência no sexo masculino de $46 \%$, também semelhante aos dados encontrados na nossa população. Observando-se a média de idade ao diagnóstico da neoplasia, percebe-se que Pinho et al. obteve uma média de idade de $57,5 \%$, assim como os demais estudos demonstrados na Tabela 1. Devido ao diagnóstico tardio dessa patologia, foi observado que a maior prevalência se encontrava no grau de invasão T3 e com ausência de linfonodos acometidos, sendo o estádio III o mais encontrado, assim como nos trabalho de Nogueira et al., Priolli et al. e Torres Neto et al. Esse fato evidencia que as políticas de saúde para a detecção de neoplasia colorretal precoce têm melhorado a sobrevida e o prognósticos dos pacientes 5 .

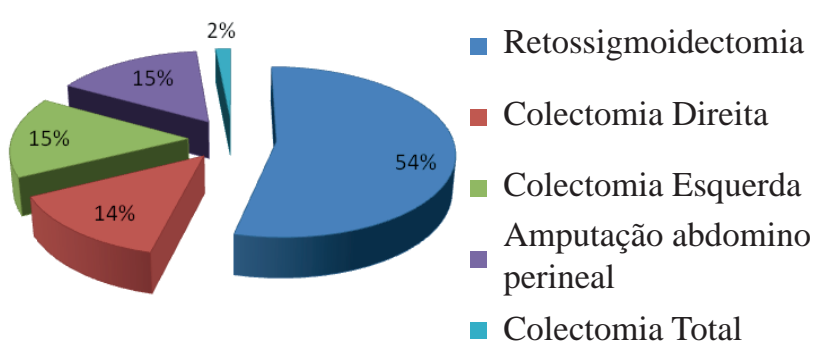

Figura 4 - Procedimento realizado

Tabela 1 - Distribuição por região

\begin{tabular}{lll}
\hline Autor, Local & $\begin{array}{c}\text { Masculino } \\
(\mathbf{\%})\end{array}$ & $\begin{array}{c}\text { Feminino } \\
(\mathbf{\%})\end{array}$ \\
\hline Cruz, Belo Horizonte & 47 & 53 \\
Neto, Sergipe & 43,3 & 56,2 \\
Pinho, Joinvile & 46 & 54 \\
Kock, Tubarão & 43,2 & 58,8 \\
Nogueira, Belo Horizonte & 37,8 & 62,2 \\
Priolli, Bragança & 45,1 & 54,9 \\
Mendes, Salvador & 46,3 & 53,7 \\
\hline
\end{tabular}


Quanto à distribuição topográfica dos cânceres colorretais, houve um predomínio na região do retossigmoide, seguido do cólon esquerdo, como demonstrado por Torres Neto et al. ${ }^{6}$. Sendo assim, a principal cirurgia realizada foi a retossigmoidectomia, fato esperado devido à distribuição da localização dos tumores.

Em relação ao grau de diferenciação tumoral, os tumores moderadamente diferenciados têm sido os mais comuns, com 50 a $60 \%$ de acometimento, seguidos dos bem diferenciados (Figura 5). Referente aos valores do CEA, a maioria da população apresentava valor menor que 3 , valor útil para o prognóstico e acompanhamento, mas não para diagnóstico de neoplasia $^{5-7}$.

\section{CONCLUSÃO}

Os dados obtidos corroboram a literatura, demonstrando que, no nosso meio, ocorre predomínio de mulheres com câncer colorretal. O pico de incidência foi na sétima década de vida, com maior incidência do adenocarcinoma moderadamente diferenciado e com seu diagnóstico tardio, o que reforça a necessidade de diagnóstico precoce.

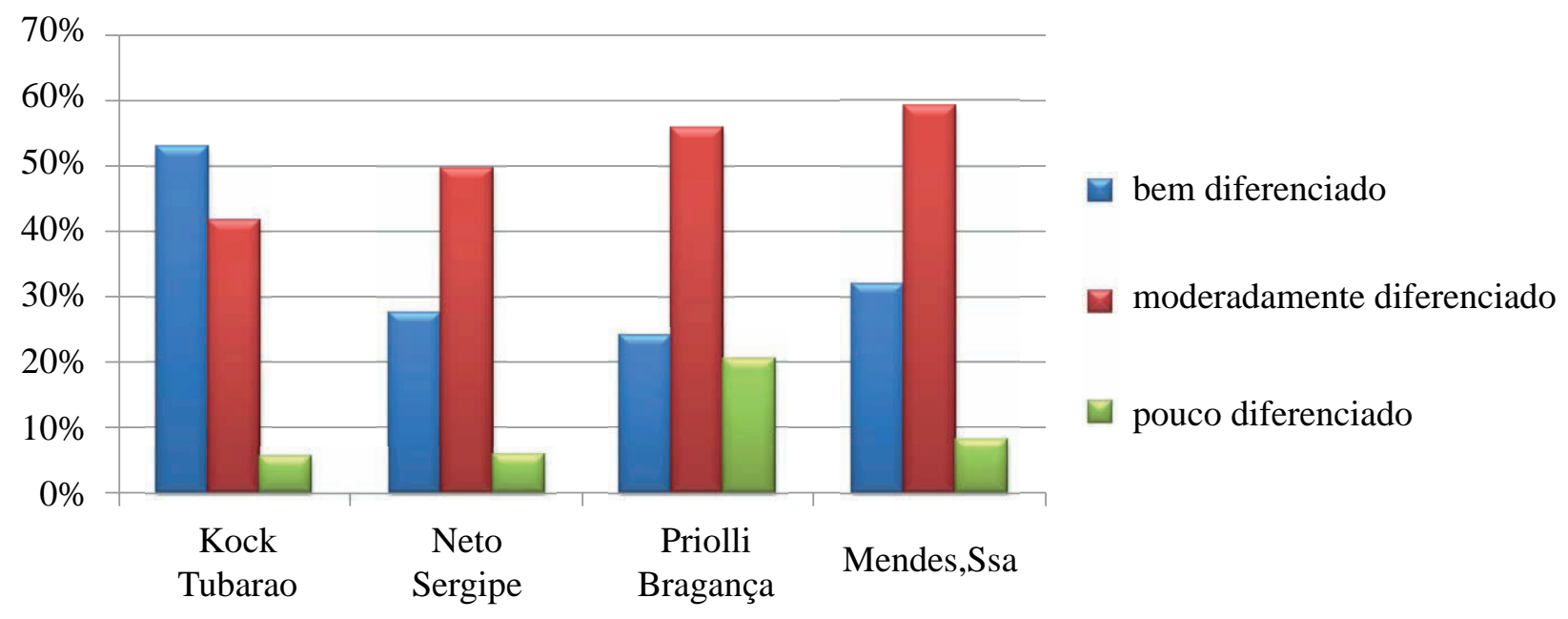

Figura 5 - Distribuição por tipo histológico

ABSTRACT: The low number of registration of colorectal cancer cases in Brazil has shown the difficulty in understanding the epidemiology of this disease, thus leading to a poor government planning for early diagnosis. The aim of this study was to report the cases of a service in Salvador during three years and compare it with the national literature data. The data were analyzed, and we found similarity to the Brazilian literature: higher incidence among females (53.7\%), mean age of 57 years at diagnosis. Stage III was observed in $40.4 \%$ of patients, and retossigmoidectomy surgery was most often performed (54\%). This study showed epidemiological characteristics of the population of Bahia, being observed late diagnosis of the disease and the need for investment in early detection.

Key works: Colorectal neoplasms/epidemiology. Neoplasms.

\section{REFERÊNCIAS}

1. Drumond CA, Ferro RAF, Nogueira AMF, Profeta da Luz MM, Conceição AS, Silva RG, et al. Câncer colorretal em pacientes com idade inferior a 30 anos. Rev Bras Coloproct. 2003;23(3):147-54.

2. Saad-Hossne R, Prado RG, Bakonyi Neto A, Lopes OS, Nascimento SM, Santos CRV, et al. Estudo retrospectivo de pacientes portadores de câncer colorretal atendidos na
Faculdade de Medicina de Botucatu no período de 20002003. Rev Bras Coloproct. 2005;25(1):31-7.

3. Pinho MSL, Fereira LC, Brigo MJK, Perreira Filho A, Wengerkievicz A, Ponath A, et al. Incidência do câncer colorretal na regional de saúde de Joinvile (SC). Rev Bras Coloproct. 2003;23(2):73-6.

4. Torres Neto JR, Teixeira FR, Prudente ACL, Silvino CJ, Arciere JS, Vieira Filho MC. Estudo demográfico do câncer cólon e reto no estado de Sergipe. Rev Bras Coloproct. 2008;28(2):215-22. 
5. Lupinacci RM, Campos FGCM, Araujo SEA, Imperiale AR, Seid VE, Habr-Gama A, et al. Análise Comparativa das características clínicas, anatomopatológicas e sobrevida entre pacientes com câncer colorretal abaixo de 40 anos de idade. Rev Bras Coloproct. 2003;23(3):155-62.

6. Simoes DL, Ramos JR, Pinheiro RR, Baptista AS. Câncer colorretal em pacientes jovens. Rev Bras Coloproct. 1987;7(4):139-41

7. Martin EW, Cooperman M, et al. Colorectal Carcinoma in patients less than 40 years of age. Dis Colon Rectun. 1981;29:322-5.

8. Carneiro Neto JD, Barreto JBP, Freitas NS, Queiroz MA. Câncer colorretal: Características clínicas e anatomopatológicas em pacientes com idade inferior a 40 anos. Rev Bras Coloproct. 2006;26(4):430-5.

9. Monteiro EP, Salen JB, Taglietti EM, Alburqueque JC, Formigas GJS. Neoplasia Colorretal até 40 anos - Experiência em cinco anos. Rev Bras Coloproct. 2006;26(2):156-61

10. Marble K, Banergee S, Greenwald L. Colorectal carcinoma in young patients. J Surg Oncol 1992;51(3):179-82.

11. Compton CC, Fielding LP, Burgart LJ, Conley B, Cooper HS, Hamilton SR, et al. Prognostic factors in colorectal cancer. College of American Pathologists Consensus Statement 1999. Arch Pathol Lab Med. 2000;124(7):97994.
12. Cruz GMG, Ferreira RMRS, Neves PM. Câncer retal: Estudo demográfico. Diagnóstico e estadiamento de 380 pacientes acompanhados ao longo de quatro décadas. Ver Bras Coloproct. 2004;24(3):208-24.

13. Espirito Santo GF, Aguilar-Nascimento JE, Kishima MO, Takiuchi A. Correlação de fatores anatomopatológicos com a sobrevida de pacientes operados por adenocarcinoma colorretal. Rev Col Bras Cir. 2008;35(3):182-7.

14. Minardi Jr AJ, Sitting KM, Zibari GB, McDonald JC. Colorectal cancer in the Young patient. Am Surg 1998;64:849-53.

15. Nelson H, Petrelli N, Carlin A, Couture J, Fleshman J, Guillem J, Miedema B, Ota D, Sargent D; National Cancer Institute Expert Panel. Guidelines 2000 for colon and rectal cancer surgery. J Natl Cancer Inst. 2001:93(8);583-96.

16. Perreira Junior T, Alves AJC, Nogueira AMMF. Câncer colorretal: análise anatomopatológica de 476 colectomias consecutivas em Belo Horizonte (MG). J Bras Patol Med Lab. 2005;41(3)175-84.

\section{Endereço para correspondência:}

Carlos Ramon Silveira Mendes

Rua São Domingos Sávio, 150 - Nazaré

CEP 40050-520 - Salvador (BA), Brasil

Tel. (71) 3241-5731 / 9971-5852

E-mail: proctoramon@hotmail.com 